\title{
ATLAS physics results (not Higgs)
}

\author{
A.R. Weidberg ${ }^{1, a}$, on behalf of the ATLAS Collaboration \\ ${ }^{1}$ Physics Department, Oxford University, U.K.
}

\begin{abstract}
These proceedings give a review of some of the non-Higgs physics results from ATLAS. The topics covered include Standard Model, exotics and SUSY. Rather than summarizing the very large number of results available in these areas, a few analyses are picked out to emphasize some important common themes for LHC physics. The first theme is the importance of good experimental resolution to maximize the sensitivity to new physics. As all new Beyond the Standard Model (BSM) physics will suffer from Standard Model backgrounds, a very good understanding of SM processes is also essential. The other two general themes that will be discussed are the choice of optimized variables and the use of data-driven background estimates.
\end{abstract}

\section{Introduction}

The main motivation for the LHC is understanding the origin of mass and the search for Beyond the Standard Model (BSM) physics. The first topic is covered elsewhere in these proceedings. In order to achieve good sensitivity in searches for BSM physics it is essential to have a very good detector performance. A very brief summary of the ATLAS detector will be given in section 2. A few BSM searches are relatively simple in that they only require good experimental performance in general and resolution in particular but they do not require a detailed understanding of Standard Model (SM) physics. For example a BSM process which generates a narrow resonance on top of a smooth SM background can be searched for using 'bump hunting' on top of a smooth background. A classic example is the $Z^{\prime}$ search [1] in the $e^{+} e^{-}$decay channel, illustrated in fig. 1. The SM background is falling smoothly above the mass of the $Z$ and a high mass sequential standard model $Z^{\prime}$ would be visible as a 'bump' above the SM background. However many other BSM physics will produce more complicated final states which will require very good understanding of the SM backgrounds. These BSM processes could involve t-channel exchange, have broad resonances or have multiple undetected particles making the difference between signals and SM backgrounds much harder to disentangle. A review of some of the measurements which result in improved knowledge of the SM are discussed in section 3. Having gained confidence in our understanding of the SM at LHC energies, in section 4 we look at a few examples of searches for exotics physics and in section 5 we discuss some example SUSY searches. More information on all subjects is contained in other papers in these proceedings. All ATLAS publications and public notes are available [2]. Finally, a summary and outlook are given in section 6.

ae-mail: t.weidberg1@physics.ox.ac.uk 


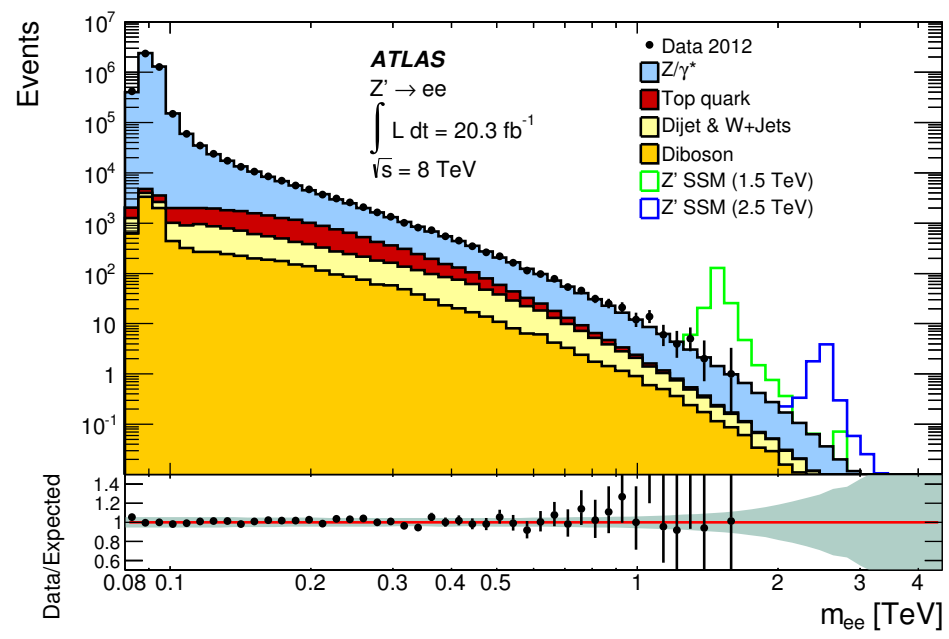

Figure 1. Dielectron invariant mass distributions after event selection, with two selected $Z^{\prime}$ SSM signals overlaid, compared to the stacked sum of all expected backgrounds, and the ratios of data to background expectation [1]. The green band in the ratio plot shows the systematic uncertainties

\section{The ATLAS detector}

The ATLAS detector is described in detail in [3] and references therein. The Inner Detector (ID) consists of silicon pixels and strip detectors as well as a transition radiation tracker (TRT). The ID is inside a $2 \mathrm{~T}$ superconducting solenoid magnet and provides precise momentum measurements of charged particles. The calorimeter systems are sampling calorimeters based on liquid argon or scintillating tiles as the active medium. There are a system of superconducting magnets outside the calorimeter which generate a toroidal magnetic field. The muon spectrometer uses different technologies to provide high precision tracking and triggering. The results presented in these proceedings rely on the excellent performance of all ATLAS detector systems.

\section{Standard Model}

This section will consider two themes in SM physics at LHC energies: improving the precision of the Parton Distribution Functions (PDF) and tuning the parton radiation in Monte Carlo generators. There are several processes which are sensitive to the PDFs and we will consider two examples. The jet cross sections are sensitive to the gluon PDF. The ratio of the jet cross sections at centre of mass energies of $\sqrt{s}=2.76 \mathrm{TeV}$ and $\sqrt{s}=7 \mathrm{TeV}$ are particularly useful because many experimental systematic uncertainties cancel in the ratio. The ratio of cross sections was measured as a function of the dimensionless quantity $x_{T}=2 p_{T} / \sqrt{s}$. The data are used in a global fit of the PDFs and the results for the gluon distribution ${ }^{1} x g(x)$ are shown [4] fig. 2. The ATLAS data help reduce the errors on $g(x)$, particularly at low and high values of $x$. The second process is charm production via $g+s \rightarrow W+c$. Therefore by identifying $c$ quarks in association with leptonic decays of the $\mathrm{W}$, we can determine constraints on the $s$ quark PDF. One way of identifying $c$ quarks is by tagging $D^{\star} \rightarrow D \pi$ decays using the small mass difference $\Delta M=M\left(D^{\star}\right)-M\left(D^{0}\right)$. The data are combined with the HERA data in a global PDF fit [5]. The resulting fit (see fig. 3) shows that the strange quark suppression is much lower than was assumed before the start of LHC and the uncertainties have been significantly reduced.

The next theme we consider for the SM analysis is measuring distributions that are sensitive to QCD parton radiation. The first example considered is the production of $W^{ \pm}$in association with

\footnotetext{
${ }^{1} x$ refers to the momentum fraction of the proton carried by a parton.
} 

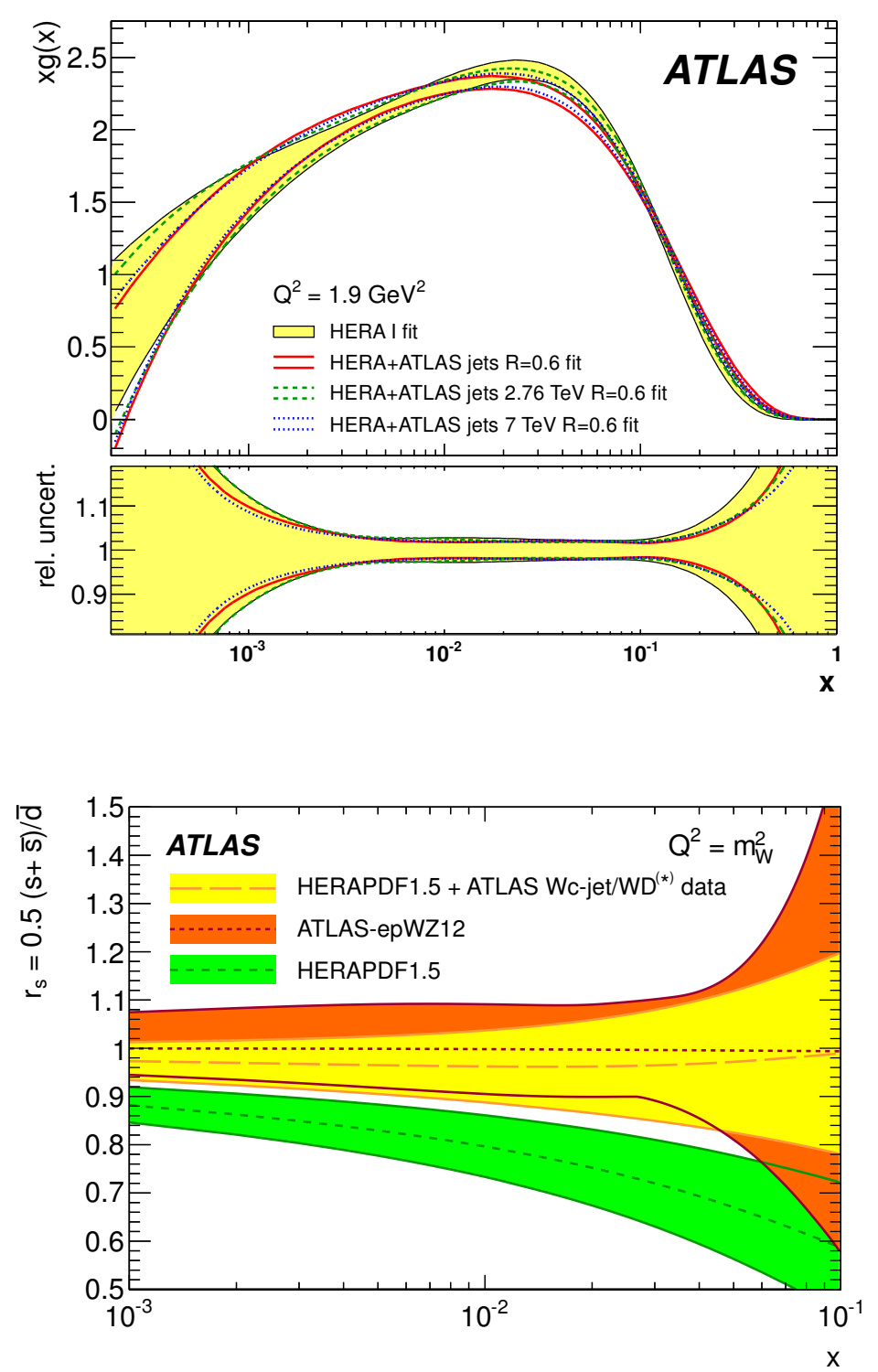

Figure 2. Momentum distributions for the gluon $x g(x)$ with their relative experimental uncertainties [4] as a function of $x$ at $Q^{2}=1.9 \mathrm{GeV}^{2}$.
Figure 3. Ratio of strange-to-down sea-quark distributions $r_{s}=0.5(s+\bar{s}) / d$ as a function of $x$ as assumed in HERAPDF1.5 PDF compared to the ratio obtained from the fit including the ATLAS $W c-j e t / W D^{(\star)}$ data [5] and the ratio obtained from ATLAS-epWZ12. The error band on the ATLAS $W c-j e t / W D^{(\star)}$ measurements represents the total uncertainty. The ratio $r_{s}$ is shown at $Q^{2}=m_{W}^{2}$.

jets [6]. The spectrum of the leading jet $p_{T}$ in these events is compared to various QCD calculations in fig. 4. There is a shape discrepancy between the predictions from BLACKHAT+SHERPA and the LoopSim Calculations compared to the data. There is reasonable agreement between the ALPGEN prediction and the data, which illustrates the importance of tuning the QCD parton radiation.

Next we consider the distribution of the transverse momentum of the $Z, p_{T}^{Z}$. At low values of $p_{T}^{Z}$ this is very sensitive to the parton radiation and in principle can be predicted by parton shower models like PYTHIA. The data [7] are compared to two PYTHIA tunes in fig. 5 and we can see that there is 

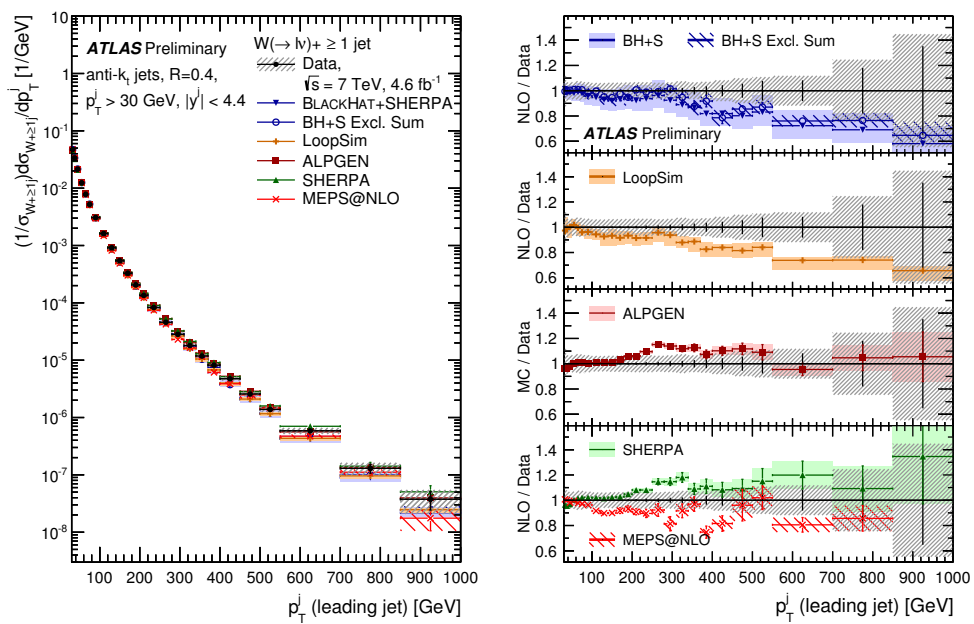

Figure 4. W+jets cross section as a function of the leading jet $p_{T}$ in $n_{\text {jets }} \geq 1$ events [6]. The left-hand figure shows the differential cross sections and the right-hand figure shows the ratios of the predictions to the data.

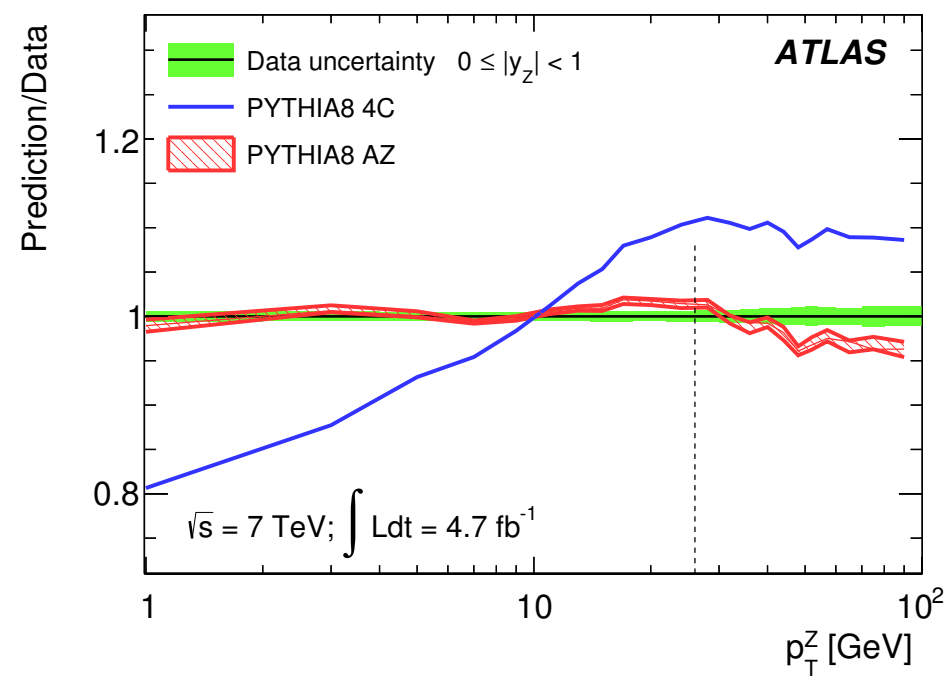

Figure 5. Comparison of tuned predictions to the measured $p_{T}^{Z}$ differential cross sections [7]. Comparison of the PYTHIA8 generator with the $4 \mathrm{C}$ and $\mathrm{AZ}$ tunes to the muon-channel $p_{T}^{Z}$ data and electron-channel $\phi^{\star}$ data. The vertical dashed lines show the upper limit of the tuning range.

good agreement if the tuning is correct but the predictions of another tune disagree very significantly with the data.

In future Higgs searches involving $H \rightarrow b \bar{b}$ decays will become important. It is therefore interesting to use $Z \rightarrow b \bar{b}$ to study the identification of a resonant signal in $b \bar{b}$ final states. The invariant mass distribution for $b \bar{b}$ pairs [8] is shown in fig. 6. A signal for $Z \rightarrow b \bar{b}$ is clearly visible above the large QCD background. This illustrates how it is possible to identify resonances on top of large backgrounds.

The final SM study we consider here is the electroweak production of $W^{ \pm} W^{ \pm}$pairs. One of the key features of the Higgs theory is that it tames the violation of unitarity that would be observed at high mass of the $W^{ \pm} W^{ \pm}$pair in the SM in the absence of a Higgs boson. Therefore studying this process at high mass is one of the critical tests of the theory. The first observation of this process has been performed using like sign Ws in order to suppress backgrounds. A clear signal above the background 

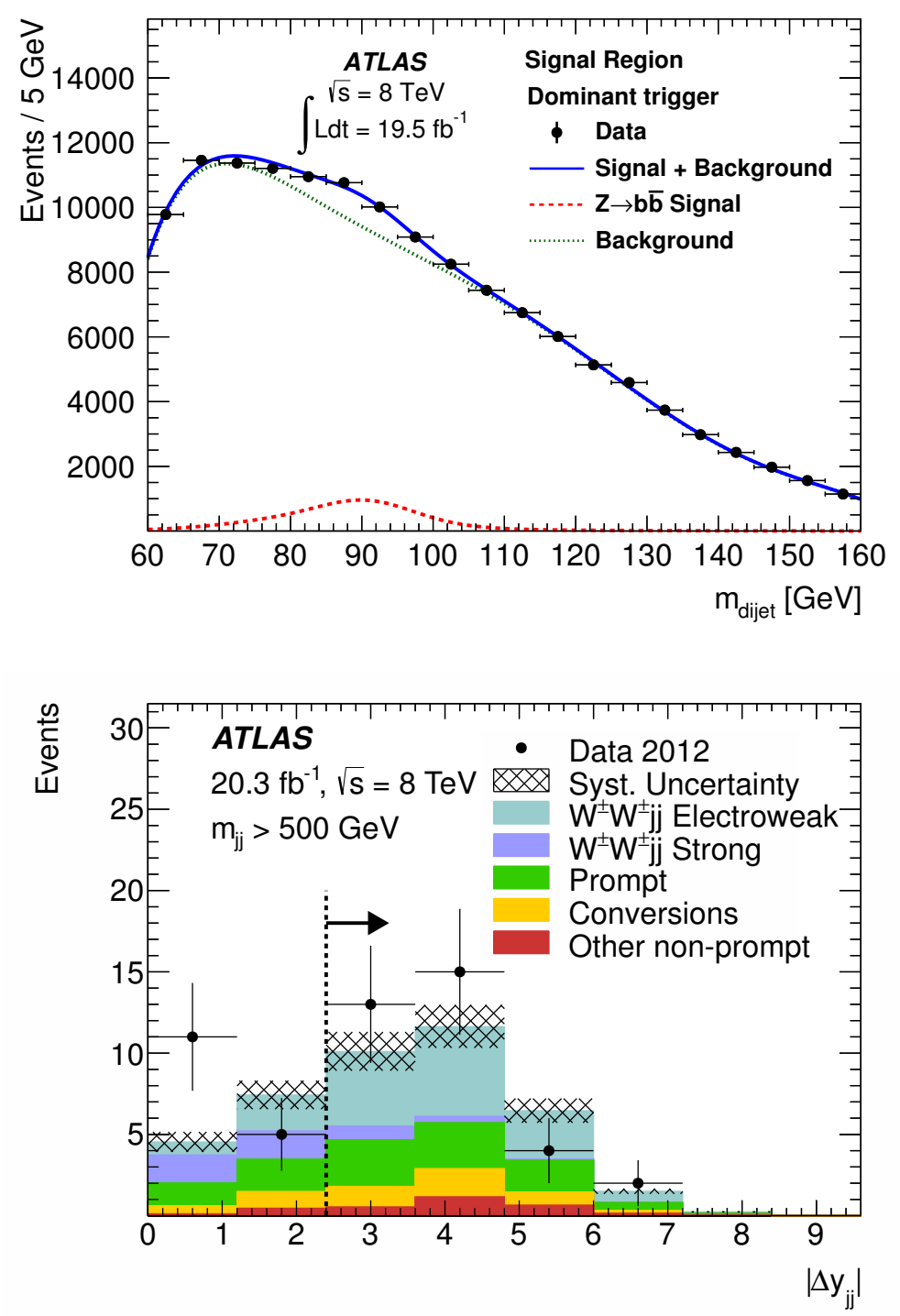

Figure 6. Invariant mass distribution for $b \bar{b}$ final states [8]. The lines represent the signal (dashed), backgrounds (dotted) and the sum of the two (solid).

Figure 7. The rapidity difference between the two highest $p_{T}$ jets in an 'inclusive' sample of two same-sign leptons plus two jets [9]. The dashed line indicates the selection designed to enhance the electroweak production process.

is seen [9] in the distribution of the rapidity gap between the two leading jets in the events as shown in fig 7.

\section{Exotics}

There are a very large number of BSM theories and there have been many different searches performed at the LHC. A particularly interesting one is the search for 'monojets' as these could be a signature for the production of WIMPs which could provide the dark matter required by astrophysical data. The distribution of missing transverse energy $\left(E_{\mathrm{T}}^{\mathrm{miss}}\right)$ from an ATLAS monojet search [10] is shown in fig. 8. There are large SM backgrounds, mainly involving $Z \rightarrow v \bar{v}$ decays. The use of datadriven background estimates allows for rather precise background calculations. There is very good 


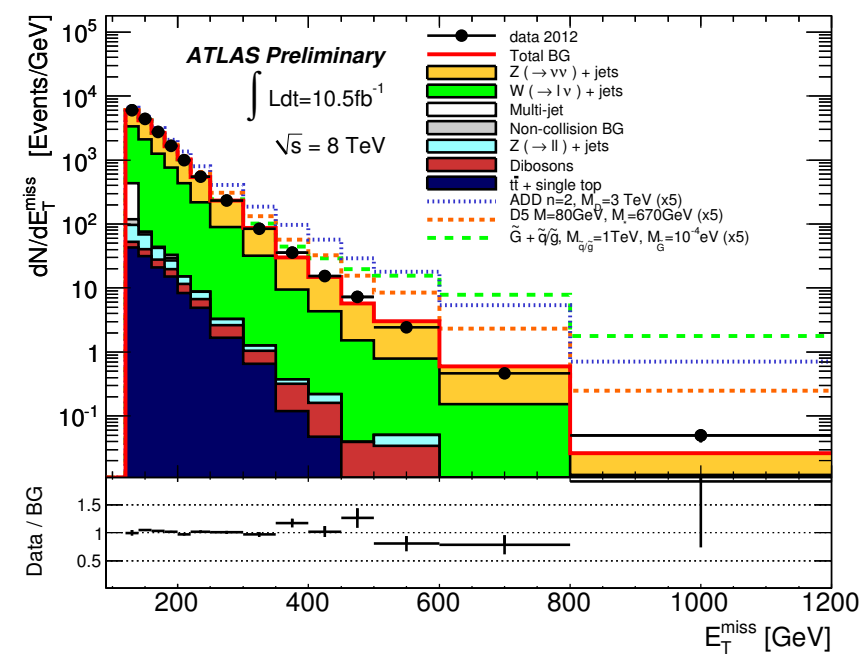

Figure 8. Measured $E_{\mathrm{T}}^{\text {miss }}$ distributions in a monojet search [10] compared to the predictions for SM backgrounds (histograms). Only statistical uncertainties are shown. For illustration purposes, the impact of different ADD, WIMP, and GMSB scenarios are included.

agreement between the data and the calculated SM backgrounds, which allows limits to be placed on several BSM theories. Similar studies can be made with other objects recoiling against $E_{\mathrm{T}}^{\text {miss }}$ ('mono$X^{\prime}$ events).

The measurements of mono-X can be compared quantitatively to the results of the direct dark matter searches using Effective Field Theories (EFT). The direct dark-matter searches are looking for the scattering of a WIMP on a quark in a nucleus and trying to detect the resulting nuclear recoil. Collider experiments can use Initial State Radiation (ISR) combined with the production of pairs of WIMPs. The WIMPs do not interact in the detector but the reaction is tagged by the large $E_{\mathrm{T}}^{\text {miss }}$ from the ISR. The results depend on which operator is assumed in the EFT and one example of such an analysis [11] using mono $\mathrm{W}$ events is shown in fig. 9. The results depend on the sign of the interference between $W$ emission from a quark and an anti-quark. However, a general feature of this analysis is the complementarity between the direct dark matter searches and the collider studies; it is very difficult for the direct dark matter searches to detect low mass WIMPs as the resulting recoil energies will be very low, whereas the collider experiments can probe this region. The direct dark matter searches have weaker limits in the case of spin-dependent interactions compared to ATLAS for which the results are similar for both spin-dependent and spin-independent interactions.

The last topic in the area of exotic physics covered is that of a search for $t \bar{t}$ resonances. Such signals are predicted in a wide class of BSM theories. Top production is also important as a background for other searches, in particular for stop squarks. The reconstruction of separate objects from $t \rightarrow b W$ decays becomes inefficient for very high $p_{T}$ tops. Therefore the use of 'boosted' objects to improve the identification efficiency for high- $P_{T}$ tops is essential [12]. The resulting $t \bar{t}$ mass spectrum is shown in fig. 10. No evidence for an excess over the SM prediction is observed and limits on BSM are derived [12]. 

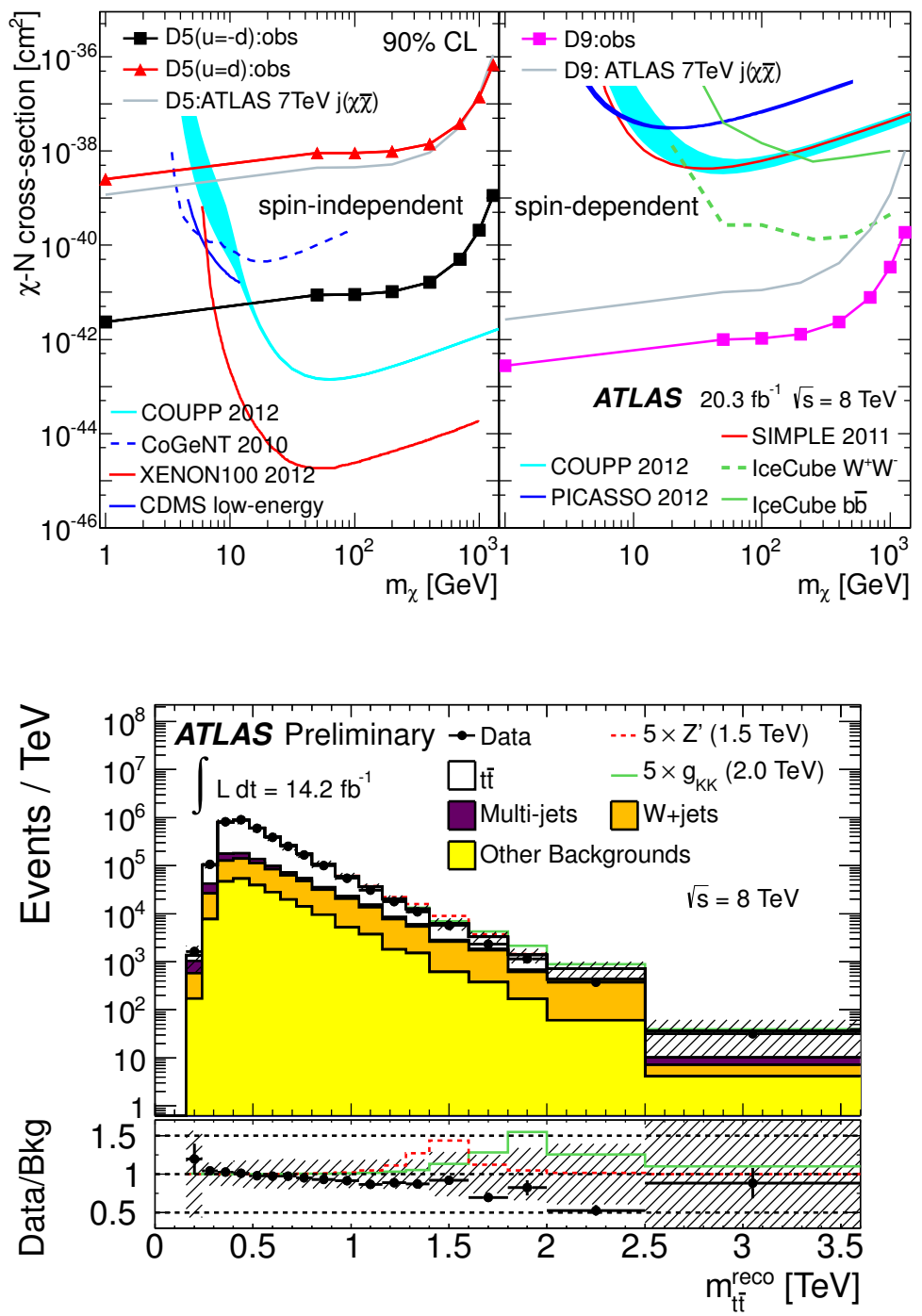

Figure 9. Limits on $\chi$-nucleon cross sections [11], compared to previous limits set by direct dark matter detection experiments and by the ATLAS $7 \mathrm{TeV}$ monojet analysis. The left hand (right hand) plot shows the limits for spin-independent (spin-dependent) scattering. All limits are shown at $90 \%$ CL.

Figure 10. The $t \bar{t}$ invariant mass spectrum, summing the spectra from the $e$ and $\mu$ channels and the two selection methods (resolved and boosted) [12]. The shaded areas indicate the total systematic uncertainties. Two benchmark signals are indicated on top of the background, a $Z^{\prime}$ with $m=1.5 \mathrm{TeV}$ and a Kaluza-Klein excitation of the gluon $g_{K K}$ with $m=2.0 \mathrm{TeV}$.

\section{SUSY}

Supersymmetry (SUSY) is an attractive extension of the SM that can explain the hierarchy problem and provides a natural candidate for dark matter. The production cross sections for different combinations at the LHC with $\sqrt{s}=8 \mathrm{TeV}$, of SUSY particles [13] are shown in fig. 11. There is a large cross section for strongly interacting squarks and gluinos but the cross section for particles produced via electroweak interactions are much smaller. The cross sections for a given type of particle decreases rapidly with the mass of the particle. The decays of SUSY particles are very model-dependent and in particular they depend on the relative masses as this determines which decay modes are kinematically accessible. It is not practical to study the full parameter space of SUSY models, therefore one approach is to use simplified models with particular decays assumed to have a branching ratio of $100 \%$. One search uses events with jets and large $E_{\mathrm{T}}^{\text {miss }}$ as this is efficient at rejecting many of the SM back- 


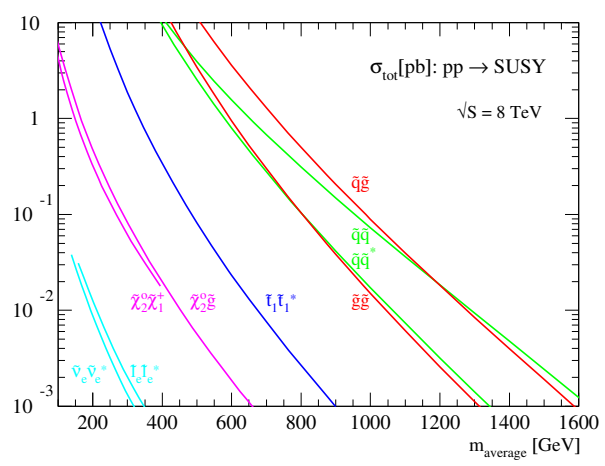

Figure 11. Cross sections for production of pairs of SUSY particles as a function of the average mass of a pair of SUSY particles.

grounds [14]. This study allows for the possibility that there is only one non-degenerate squark, which results in much lower cross sections than the assumption of their being 8 non-degenerate squarks. This therefore requires a very good understanding of the SM backgrounds, which is done using a careful choice of control regions (CR), validation regions (VR) and signal regions (SR). An example is the $\gamma+$ jets sample which is used as a CR to model the distribution of $E_{\mathrm{T}}^{\text {miss }}$ generated by $Z+j e t s$ with $Z \rightarrow \bar{v} v$ decays. A very useful variable to distinguish the signal from the background is the effective mass

$$
m_{\mathrm{eff}}=E_{\mathrm{T}}^{\mathrm{miss}}+\sum p_{T}^{i}(j e t)
$$

where the sum runs over jets with $p_{T}>40 \mathrm{GeV}$. The distribution of $m_{\mathrm{eff}}$ for this CR is shown in fig. 12. The distribution of $m_{\mathrm{eff}}$ for the two-jet signal region is shown in fig. 13. In the absence of a signal, limits can be placed in the plane of the mass of the squark and the mass of the neutralino, assuming that the gluinos are decoupled and that the squark decays with $100 \%$ BR as $\tilde{q} \rightarrow q \chi_{1}^{0}$ and the results are shown in fig. 14. Much stronger limits are obtained with the assumption of 8 nondegenerate squarks, but useful limits are also obtained with the pessimistic assumption that there is only one non-degenerate squark.

The hierarchy argument strongly favours the existence of light third generation squarks, therefore the search for sbottom and stop squarks is particularly important. One search looks for the production of $\tilde{b} \tilde{b}$ pair with the subsequent decay $\tilde{b} \rightarrow b \chi_{1}^{0}$ and the resulting signatures is two b-jets and large $E_{\mathrm{T}}^{\text {miss }}$. The main background from $t \bar{t}$ production is suppressed using a requirement on the invariant mass of the $b \bar{b}$ pair, $m_{b \bar{b}}>200 \mathrm{GeV}$. No excess above the SM background is observed and the resulting limits [15] in terms of the mass of $\tilde{b}$ and the mass of the $\chi_{1}^{0}$ are shown in fig. 15.

One particularly powerful search for stop squarks was performed in the 1-lepton + jets $+E_{\mathrm{T}}^{\text {miss }}$ channel. The $\overline{\tilde{t}}$ pairs are produced strongly and the resulting decay modes of the stop depend on the mass difference $\Delta m=m_{\tilde{t}}-m_{\chi_{1}^{0}}$. If $\Delta m>m_{t}$ then the stop decays via $t \rightarrow \tilde{t} \chi_{1}^{0}$, with the subsequent SM decays of the top. If $\Delta m<m_{W}+m_{b}$ then the stop decays directly as $\tilde{t} \rightarrow b W \chi_{1}^{0}$. Different signal regions are optimized for these combinations. The resulting limits [16] in the mass plane of the stop $(\tilde{t})$ and the neutralino $\left(\chi_{1}^{0}\right)$ are shown in fig. 16. The region for which $m_{\tilde{t} 1}$ is slightly greater than $m_{t}+m_{\chi_{1}^{0}}$ is difficult to exclude because the kinematics are very similar to the SM $t \bar{t}$ production. However some of this region can be excluded by performing a precision measurement of the $\bar{t} t$ production cross section as stop pair production would enhance the measured cross section. The $t \bar{t}$ production cross section is 

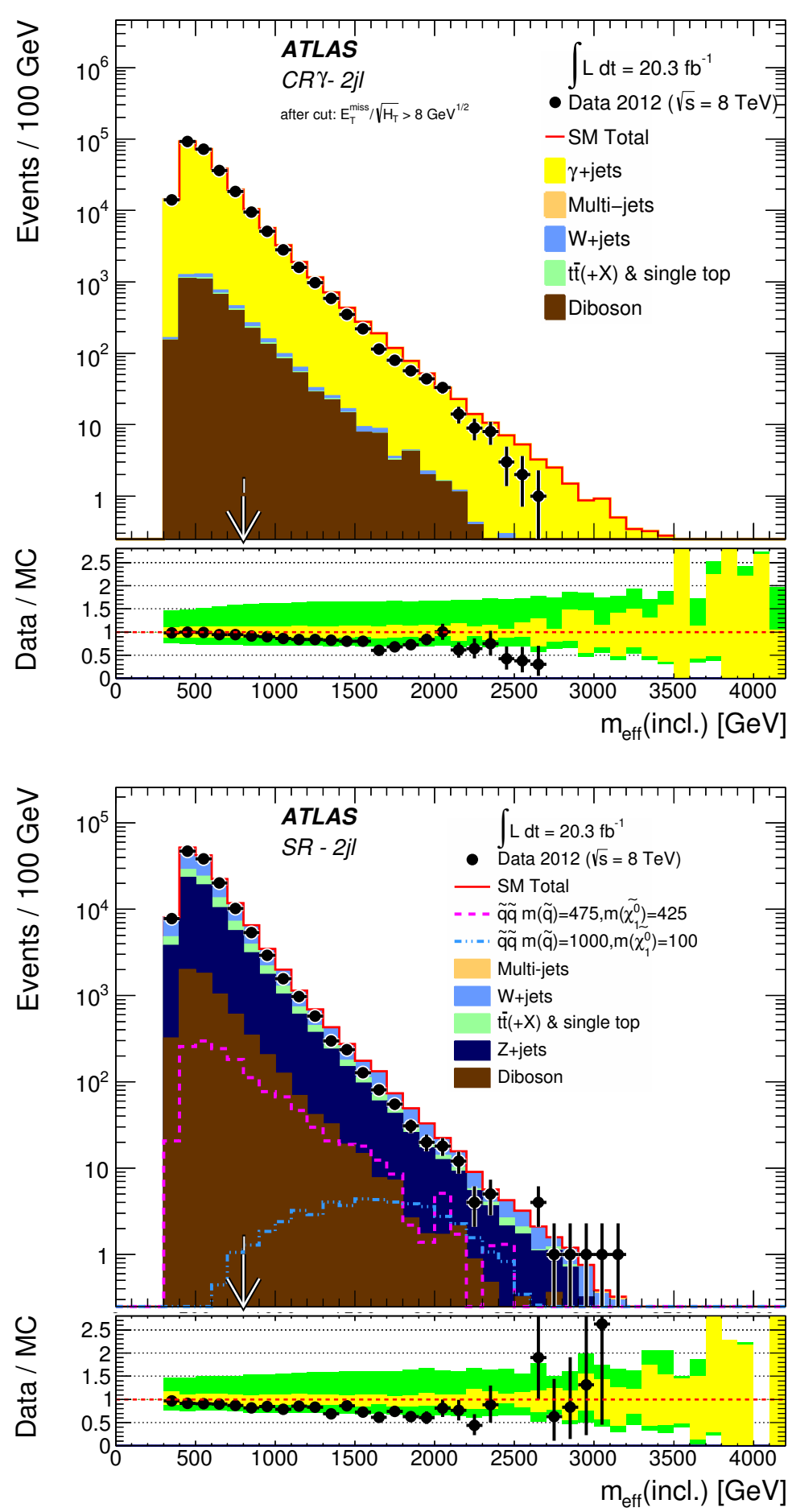

Figure 12. Observed $m_{\text {eff }}$ distributions in the $\gamma$ jet CR [14]. In the lower panel the light (yellow) error bands denote the experimental and MC statistical uncertainties, while the medium dark (green) bands include also the theoretical modeling uncertainty. The arrows indicate the values at which the requirements on $m_{\text {eff }}$ are applied.

Figure 13. Observed $m_{\text {eff }}$ distributions for the 2-jet signal regions [14]. In the lower panels the light (yellow) error bands denote the experimental and MC statistical uncertainties, while the medium dark (green) bands include also the theoretical modeling uncertainty. The arrows indicate the values at which the requirements on $m_{\text {eff }}$ are applied. Expected distributions for benchmark model points are also shown for comparison (masses in $\mathrm{GeV}$ ). 


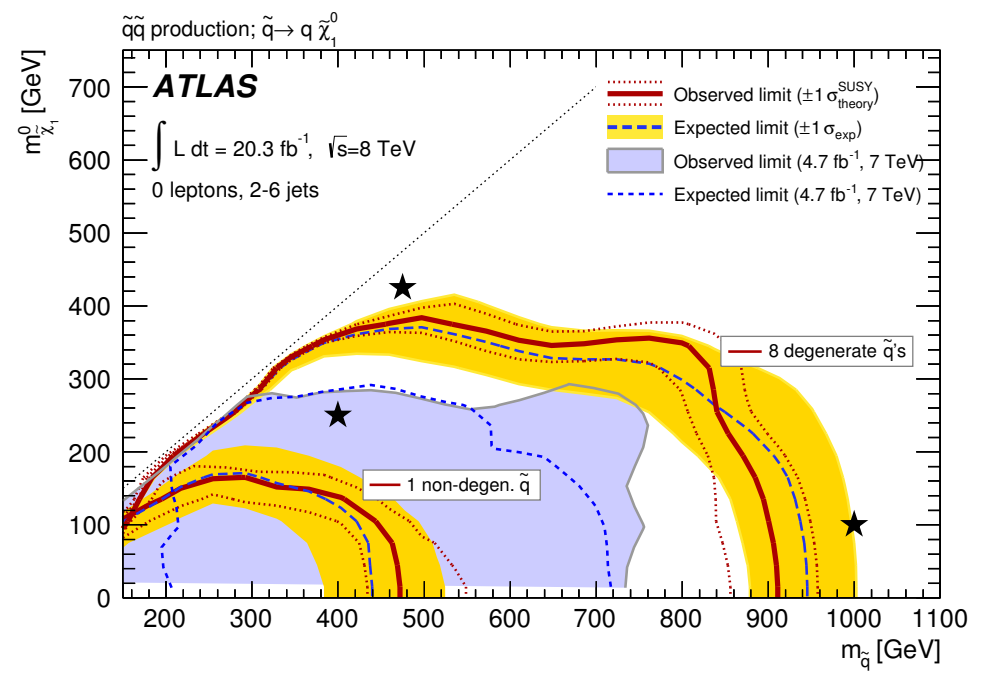

Figure 14. Exclusion limits for direct production of light-flavour squark pairs with decoupled gluinos [14].

Light-flavour squarks are required to decay to one quark and a neutralino LSP. Limits are shown for scenarios with eight degenerate light-flavour squarks (squark L+squark R), or with only one non-degenerate light-flavour squark produced. Exclusion limits are obtained by using the signal region with the best expected sensitivity at each point. The blue dashed lines show the expected limits at 95\% CL, with the light (yellow) bands indicating the $1 \sigma$ excursions due to experimental and background-only theory uncertainties. Observed limits are indicated by medium dark (maroon) curves, where the solid contour represents the nominal limit, and the dotted lines are obtained by varying the signal cross-section by the renormalization and factorization scale and PDF uncertainties.

shown from different ATLAS measurements [17] at $\sqrt{s}=7 \mathrm{TeV}$ and $\sqrt{s}=8 \mathrm{TeV}$ in fig 17. The data are consistent with the SM and the good precision allows an exclusion at the $95 \%$ confidence level to be placed on top squarks with masses between the top quark mass and $177 \mathrm{GeV}$. 

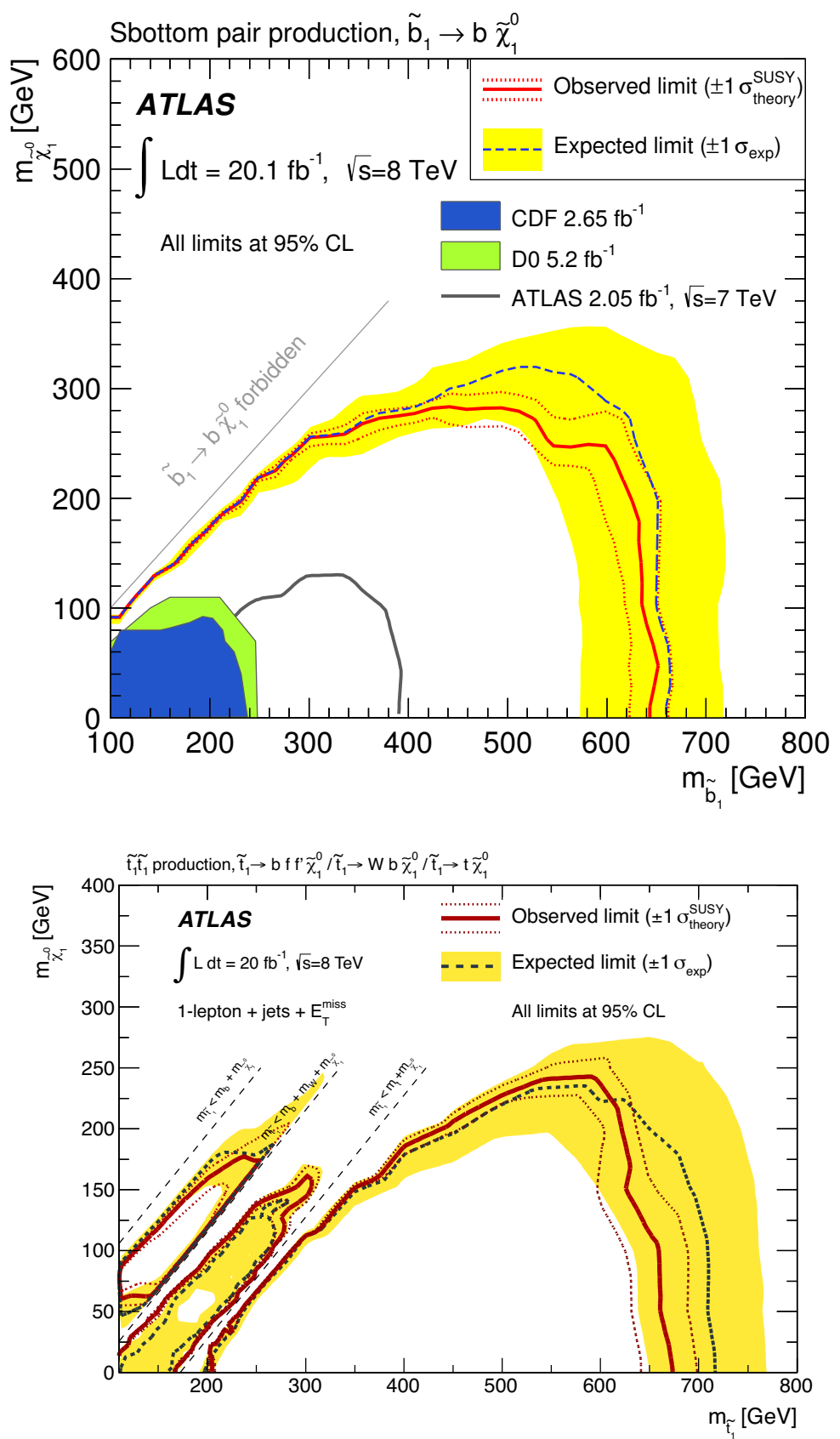

Figure 15. Expected and observed exclusion limits at 95\% CL in the $\left(m_{b 1}, m_{\chi_{1}^{0}}\right)$ mass plane for the sbottom pair production scenario considered [15]. The dashed (solid) lines show the expected (observed) limits, including all uncertainties except for the theoretical signal cross-section uncertainty (PDF and scale). The bands around the expected limits show the $\pm 1 \sigma$ uncertainties. The dotted lines around the observed limits represent the results obtained when moving the nominal signal cross section up or down by the $\pm 1 \sigma$ theoretical uncertainty.

Figure 16. Expected (black dashed) and observed (red solid) 95\% CL excluded region in the plane of $m_{t 1}, m_{\chi_{1}^{0}}$, assuming $B R\left(t_{1} \rightarrow t \chi_{1}^{0}\right)=100 \%$ [16]. 


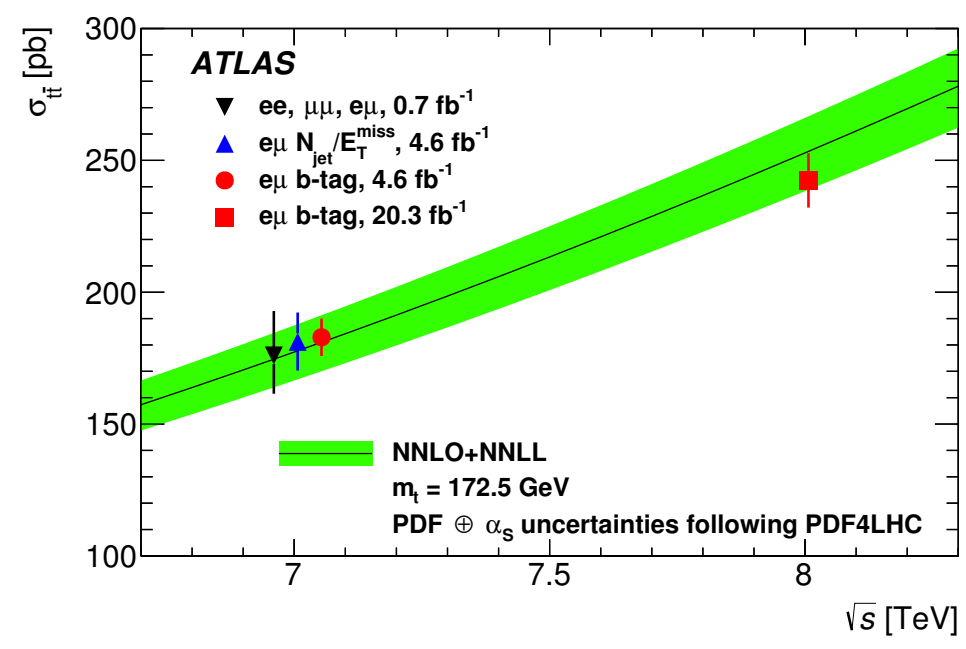

Figure 17. Measurements of the $t \bar{t}$ cross-section at $\sqrt{s}=7 \mathrm{TeV}$ and $\sqrt{s}=8 \mathrm{TeV}$ ( $e \mu$ b-tag) [17] together with previous ATLAS results at $\sqrt{s}=7 \mathrm{TeV}$ using the $e e, e \mu$ and $\mu \mu$ channels.

\section{Summary and Outlook}

Many SM studies have been carried out which have been used to improve the precision of QCD predictions. This has allowed ATLAS to perform many sensitive searches for BSM physics. Although no evidence for any BSM physics has been found, stringent limits have been placed on many models. Using effective field theories, the ATLAS 'mono- $X$ ' searches have been related to the results of the direct dark-matter searches. The resulting ATLAS limits are powerful and somewhat complimentary to those from direct dark-matter searches. The search for SUSY has continued and more difficult scenarios have been considered and powerful limits have been obtained for the third generation squarks. More details are given in other ATLAS papers at this conference and a full list of public results are available [2]. The LHC run 2 at the higher centre of mass energy will greatly open up the phase space for the discovery of BSM physics.

\section{References}

[1] ATLAS Collaboration, arXiv:1405.4123 [hep-ex], accepted by Phys. Rev. D.

[2] ATLAS public results available at https://twiki.cern.ch/twiki/bin/view/AtlasPublic/WebHome.

[3] ATLAS Collaboration, JINST 3 (2008) S08003.

[4] ATLAS Collaboration, EPJC (2013) 732509.

[5] ATLAS Collaboration, JHEP05(2014)068.

[6] ATLAS Collaboration, ATLAS-CONF-2014-035, http://cds.cern.ch/record/1735193.

[7] ATLAS Collaboration, arXiv:1406.3660 [hep-ex], submitted to JHEP.

[8] ATLAS Collaboration, arXiv:1406.3660 [hep-ex], submitted to Phys. Let. B.

[9] ATLAS Collaboration, arXiv:1405.6241 [hep-ex], accepted by Phys. Rev. Lett.

[10] ATLAS Collaboration, ATLAS-CONF-2012-147, http://cds.cern.ch/record/1493486

[11] ATLAS Collaboration, PRL 112, 041802 (2014).

[12] ATLAS Collaboration, ATLAS-CONF-2013-052 http://cds.cern.ch/record/1547568.

[13] www.thphys.uni-heidelberg.de/ plehn/includes/prospino/prospino_lhc8.eps.

[14] ATLAS Collaboration, arXiv:1405.7875, submitted to JHEP. 
ICNFP 2014

[15] ATLAS Collaboration, JHEP10(2013)189.

[16] ATLAS Collaboration, arXiv:1407.0583, submitted to JHEP.

[17] ATLAS Collaboration, arXiv:1406.5375, Submitted to: Eur. Phys. J. C. 